\title{
An annotated check-list of British Pleistocene, Holocene and modern freshwater ostracods
}

\author{
H. I. GRIFFITHS ${ }^{1}$ \& J. G. EVANS ${ }^{2}$ \\ 'Department of Genetics, School of Biological Sciences, University of Leeds, Woodhouse Road, Leeds LS2 9JT, UK. \\ ${ }^{2}$ School of History \& Archaeology, University of Wales College of Cardiff, PO Box 909, Cardiff CF1 3XU, UK.
}

\begin{abstract}
A revised check-list of British Pleistocene, Holocene and Recent freshwater ostracods is given, and notes provided on the taxonomy and occurrence of some rare and newly-reported species. Stenocypria fischeri (Lilljeborg. 1883) and the hypogean species Pseudocandona cf. eremita (Vedjvosky, 1882) and Pseudocandona breuili (Paris, 1920) are reported from Britain for the first time. J. Micropalaeontol. 14(1): 59-65. April 1995.
\end{abstract}

\section{INTRODUCTION}

Over recent years there has been renewed interest in freshwater ostracods, particularly because of their potential in palaeolimnological and palaeohydrological studies (see reviews by Delorme, 1989; De Deckker \& Forester, 1988: Carbonel et al. 1988; Holmes, in press). This increase in ecological interest has been accompanied by a spate of taxonomic and systematic research that has done much to increase our understanding of evolutionary patterns and processes in freshwater Ostracoda. Some freshwater ostracod lineages are evolutionarily dynamic, and divergence within the Holocene has been demonstrated for at least one cluster of lake-specific taxa (Martens, 1990). It is possible that the examination of ostracod valves in extended lake cores (which often cover periods of several thousands of years) may prove to be a powerful tool to the evolutionary biologist (Evans \& Griffiths, 1993). However, advances in ostracod palaeobiology do require continuity in systematic nomenclature.

\section{A BRIEF HISTORY OF BRITISH OSTRACOD STUDIES}

Some of the key works in ostracod taxonomy were based on studies of the British and Irish faunas during the last century, notably through the work of G. S. Brady and colleagues (e.g. Brady \& Robertson, 1869; Brady \& Norman, 1889; Brady, 1910). As a result, comparatively comprehensive check-lists of the faunas of both Britain (Scourfield, 1904) and Ireland (Norman, 1905) were available within the first years of this century. Subsequently, interest in native ostracod faunas waned, and scientific attention was turned to the description of species from overseas. Some workers continued to study British freshwater species, notably P. F. Holmes, A. G. Lowndes, G. Fryer and H. M. Fox, however most had a broad interest in freshwater Crustacea, rather than a specific interest in ostracods. Hence, prior to the 1980 s, there were remarkably few published contributions, and no substantial body of work accrued. Despite this, J. E. Robinson's Pleistocene and Holocene studies continued to document ostracod faunas, as occasionally have archaeological (e.g. Griffiths \& Mount, 1993) and modern synecological works (e.g. Ham, 1982).

An updated check-list of freshwater Ostracoda was made as part of the Institute of Terrestrial Ecology's attempt to compile a complete listing of British freshwater animals (Maitland, 1977). This was not based upon the work of a single individual, but gleaned from a variety of sources, largely without reappraisal. More recently, a new faunal work on modern British freshwater ostracods has been published as part of the Linnean Society's 'Synopses of the British Fauna' series (Henderson, 1990). Unfortunately, this important benchmark was delayed in press for almost five years (Henderson, pers. comm.) and, as a result, over $30 \%$ of the specific and generic nomenclature used by Henderson had been superseded by the time of publication. The undesirable result is a loss of continuity with current European taxonomic usage (e.g. Wouters, 1989; Meisch et al. 1990). With the increasing interest in the group by both palaeoecologists and freshwater biologists, it seems timely to provide a check-list of the British species that includes not only recent revisions, but also records of Recent species omitted by Henderson (1990), plus a small number of species that are thus far known only from Pleistocene and Holocene contexts.

\section{NOTES ON SOME OF THE SPECIES}

Changes of generic assignation and specific nomenclature have come about as the result of formal revisions, the details of which are beyond the scope of this account. Key works include those of Broodbakker (1983), Carbonnel (1965), Colin \& Danielopol (1980), Danielopol (1978), Danielopol \& McKenzie (1977), Danielopol et al. (1989), Marmonier et al. (1989), Martens (1989, 1992) and Meisch $(1984,1985$. 1991). Some species do require additional comment, however.

Cypridopsis bamberi was originally described from a spring in Cornwall by Henderson (1986). Professor T. K. Petkovski (pers. comm.) initially suggested the possible 
synonymy of $C$. bamberi with $C$. brincki Petkovski, 1963, described from wells in the Azores and also known from Germany, Portugal and Macedonia (Petkovski, 1963). We examined type material of $C$. bamberi lodged in the collections of the Natural History Museum in London (BMNH) and reached a similar conclusion. More recently, Petkovski et al. (1993) have shown C. brincki to be a junior synonym of C. lusatica Shäffer, 1943, and the synonymy of $C$. bamberi has been confirmed by Dr. C. Meisch of the Natural History Museum of Luxemburg (Meisch, pers. comm.).

Eucypris anglica was posthumously described as a British endemic by Fox (1967) from sites in Buckinghamshire and Hertfordshire, but has since only been reported as a single specimen from Hampshire (Ham, 1982). Syntypes are maintained in the BM(NH)'s Fox Collection (accession nos 1967.4.3.1,2,3,4), the material consisting of four sealed microscope slides bearing valves, two intact individuals, and intact specimens decalcified in potassium hydroxide. All are mounted in glycerine jelly or euparal. These specimens compare well with illustrated descriptions of $E$. crassa in Klie (1938) and Sywula (1974), although are a little larger than usually cited (i.e. $2.2 \mathrm{~mm}$ ). At our request, Professor T. $\mathrm{K}$. Petkovski has re-examined material of $E$. anglica originally sent to him by Fox, and concluded that the species is indeed distinct. We therefore maintain E. anglica on the British list, although reinvestigation of new material of this species would be welcome.

Ilyocypris biplicata is well-known in Quaternary palaeontology, and has been recorded in the modern faunas of Canada (Delorme, 1970) and France (Meisch et al., 1990). The status of the species has long been the source of debate; Scourfield (1904) stated that I. biplicata was common in Britain, although it seems possible that he may have been confused between I. biplicata and I. gibba. Sywula (1974) lists $I$. biplicata as a subspecies of $I$. gibba. Palaeontologists have long accepted I. biplicata (sensu Diebel \& Pietrzeniuk, 1969: pl 7, figs 1-3), and Van Harten (1979) provides a valve-based diagnosis of the species. Ilyocypris biplicata is a bland ilyocypridid, in which surface ornamentation is reduced. The carapace is sub-rectangular, posteriorally and anteriorally rounded, and with ventral and posterior margins running almost parallel. In Britain the species has often been recorded at Pleistocene sites, and we have material from Holocene deposits at West Overton in Wiltshire (Griffiths \& Mount, 1993). As soft-part diagnosis remains elusive (Meisch, 1988), it would seem that $I$. gibba and $I$. biplicata would benefit from examination by either molecular or genitalia-based taxonomic techniques (cf. Martens, 1991). Until such a time as they can either be synonymized or verified, it seems prudent to maintain $I$. biplicata as a valid species.

Western European species of Potamocypris have been extensively revised by Meisch $(1984,1985)$ and the nomenclature used here differs somewhat from that of Henderson (1990). Potamocypris arcuata was first recorded in the modern British fauna by Griffiths \& Evans (1992) from a temporary, groundwater-fed pool in Hampshire. Since that time we have also found the species amongst air-dried material from Regent's Park Lake, London (leg. J. E. Robinson). The species is also known from British late Devensian and Holocene deposits as P. maculata Alm, 1914 (see list of synonyms in Meisch, 1985).

Eucypris elliptica. This is one of Britain's less well-known species; Henderson (1990) knew of no definite locations for E. elliptica in the UK. In December 1990, we collected two females from the shallows of Llangorse Lake in the Brecon Beacons National Park, Wales, and Professor D. D. Williams has recently provided further specimens collected from the Island of Bardsey off the North Wales coast.

Eucypris lilljeborgi. Again, Henderson (1990) knew of no definite records for this 'exceedingly rare' species, although $E$. cf. lilljeborgi had been reported from Holocene tufas (Preece \& Robinson, 1984; Willing, 1985). We have since found $E$. lilljeborgi living in great numbers in a run-off and rain-fed, grassy, seasonally-inundated meadow adjacent to a Phragmites-rich pond at Thornhill in Cardiff. Eucypris lilljeborgi first appears when the habitat is inundated in October or November, and then seems to breed continuously until the site dries out in early summer. The species rarely occurs in the reed beds which border the inundated grasses, and males are absent. Carapace length is more variable than usually believed; we have collected gravid females ranging from $1.42-1.88 \mathrm{~mm}$ long, although clearly identifiable as E. lilljeborgi on the basis of soft-part anatomy.

Trajancypris serrata. There has been considerable confusion over the taxonomic status of denticulate eucypridines. Some are assignable to Prionocypris serrata (Norman, 1861) of which we have modern and Holocene material from the Test Valley, Hampshire. Martens (1989) erected genus Trajancypris to accommodate subclavate eucypridines with pronounced lists and selvages on the anterior inner margins, including Eucypris serrata G. W. Müller, 1900 and E. clavata, the latter having often been recorded from the Pleistocene as Sclerocypris? clavata prisca Diebel \& Pietrzeniuk, 1969. Because of the confusion over nomenclature, it now seems almost impossible to say with confidence which denticulate eucypridine was actually meant by many earlier authors. Hence, we provisionally maintain $T$. serrata in the British check-list, despite having seen neither subfossil or modern material.

Paralimnocythere spp. There has been some confusion over the correct specific assignation of non-Balkan species of Paralimnocythere, now resolved by Martens (1992). Subfossil British material has usually been referred to $P$. compressa or $P$. cf. diebeli. Paralimnocythere compressa is known from Mid Pleistocene, late Devensian and Holocene deposits in Britain, although it has not been collected alive for over a century (Martens, 1992). Paralimnocythere diebeli was originally described from Macedonia (Petkovski, 1969) and a variant, Paralimnocythere cf. diebeli, was described from the German Mid Pleistocene (Diebel \& Pietrzeniuk, 1978: fig.2, pl. 52, figs 8-11). Paralimnocythere cf. diebeli has been reported from late Devensian deposits at Kildale, Yorkshire (Keen et al., 1984). Paralimnocythere relicta is only known from the 
modern fauna, having been reported from Hampshire by Henderson (1990).

Fabaeformiscandona siliquosa. This is an unusual species which appears to be a British endemic. Some authors have doubted this; Niichterlein (1969: 246) believed $F$. siliquosa to be synonymous with $F$. caudata. Henderson (1990) maintains $F$. siliquosa as a valid species, listing several sites in the New Forest of Hampshire. We have also collected $F$. siliquosa from permanent ponds in the New Forest and compared it with specimens of $F$. caudata from France (leg. P. Marmonier). The two species appear very different, and we have retained them both as distinct species.

Candona lactea was reviewed by Brady (1910) and retained as a valid species by Henderson (1990). Although we have not examined type material of this taxon, we have collected material that appears similar to $C$. lactea, but have never encountered mature individuals. We therefore maintain C. lactea in the British list until formal revision is made, but believe that it may be a synonym mistakenly erected upon juvenile material of another species. Further investigation is required to validate or deny this suggestion.

Pseudocandona elongata was initially described from Lakes Windermere and Ohrid (Holmes, 1937) although no type material was nominated, and none has been located (Henderson, pers. comm.). Petkovski (pers. comm.) has failed to record the species despite many years collecting at Lake Ohrid. Within recent years $P$. elongata has been reported from Lake Windermere by Horne et al. (1990), but subsequent collections have failed to provide further specimens (Horne, pers. comm.). This species also requires further investigation before it can be validated.

\section{PLEISTOCENE AND HOLOCENE SPECIES}

In addition to a diverse modern British fauna, a small number of species are known exclusively from Pleistocene and Holocene deposits, although some exist only as single records. These include Leucocythere baltica (as Limnocythere baltica), Limnocythere falcata, Limnocythere stationis, Limnocythere cf. usenensis, Ilyocypris quinculminata, I. papillata, I. schwarzbachi, Candona levanderi, Candona lozeki, Candona tricicatricosa, Fabaeformiscandona fabella (as Candona fabella), Fabaeformiscandona balatonica (as Candona balatonica), Pseudocandona breuili, Pseudocandona cf. eremita, Nannocandona faba, Scottia browniana, S. tumida, Eucypris dulcifrons, E. heinrichi, Herpetocypris ehringsdorfensis and Stenocypria fischeri. In addition, the Tertiary species Eucypris cf. gemella has been reported from Pleistocene deposits at Holderness (Catt \& Penny, 1962) and a few taxa, e.g. Candona brevicornis Klie, 1925 have been reported from the Irish Republic (Preece et al., 1986). Some taxa are believed to have stratigraphic value (Robinson, 1978), notably: Candona tricicatricosa, C. lozeki, Limnocythere falcata, Leucocythere baltica, Ilyocypris quinculminata, I. schwarzbachi, and possibly I. papillata (see Robinson, 1990). The biostratigraphic importance of Scottia spp. has been discussed in detail by Kempf (1971).
Candona lozeki has been reported from the British Holocene (Willing, 1985; Mount, 1991) and from the Mid Devensian (Gibbard et al., 1981), whilst locations for $C$. tricicatricosa are all Mid Pleistocene. Fuhrmann (1991) has suggested that $C$. lozekiC. tricicatricosa are synonymous. If this is the case, this would remove the stratigraphic value of both taxa.

Pseudocandona breuili was first described from a cave in Spain (Paris, 1920) and, as Candona breuili, is known from the German Quaternary (Diebel \& Pietrzeniuk, 1984) and the Belgian Holocene (Van Frausum \& Wouters, 1990). Definitive determination of this species is difficult without soft-parts, although we have collected it in considerable numbers from Holocene deposits at West Overton, Wiltshire and from the Test Valley, Hampshire. It appears that the species lived interstitially, and Danielopol (1978) lists $P$. breuili as a hypogean species. In some cases at least, it seems that $P$. breuili has been erroneously identified as the juvenile moult stages of Psychrodromus olivaceus.

Pseudocandona eremita is one of a cluster of hypogean ostracods that display a high degree of local endemism, and represent a distinct lineage within Pseudocandona (Danielopol, 1982). These are difficult to identify with precision without soft parts, hence we have cited our taxon as $P$. cf. eremita. The species occurs in Holocene sediments from West Overton, Wiltshire and Bossington, Hampshire, where it seems to have existed interstitially. Pseudocandona eremita has a distinctive triangulate carapace, quite unlike any other British candonid.

Stenocypria fischeri is only known in Britain from Holocene material from West Overton, Wiltshire (Griffiths \& Mount, 1993). Stenocypria fischeri is illustrated in several European faunal works (e.g. Klie, 1938: 124, figs 416-418; Sywula, 1974: 211, fig. 105, pls $17 \mathrm{~g}-\mathrm{h}$ ).

Nannocandona faba. The absence of this species from the fauna of Modern mainland Britain is rather puzzling; the species is quite widespread in Pleistocene and Holocene deposits, furthermore Nannocandona sp. has been reported from Modern Ireland (Douglas \& Healey, 1991). As Nannocandona faba often occurs in interstitial contexts, especially in rivers (Marmonier \& Danielopol, 1988 ), it is possible that it may yet be found in modern Britain, where interstitial habitats remain largely unexplored.

\section{SYSTEMATIC CHECK-LIST}

In the following species list, higher-level systematic nomenclature follows Bowman \& Abele (1982), and familial nomenclature largely conforms to Hartmann \& Puri (1974). The nomenclature of rankings below the familial level conforms to current European usage (Meisch et al., 1990). Taxonomic authorities are drawn from Kempf's index (1980a, b). Species are broadly provenanced by the following superscripts: $\mathbf{P}=$ Pleistocene, $\mathrm{H}=$ Holocene, $\mathrm{R}=$ Recent. Those taxa which have been recorded in the British fauna, but whose status is here considered questionable, are prefixed by a question mark. Cyprideis torosa is included, although more typically a species of brackish waters. 
Phylum or sub-phylum Crustacea Pennant, 1777 Class Ostracoda Latreille, 1806 Sub-class Podocopa G. W. Müller, 1894 Order Podocopida Sars, 1866 Sub-order Podocopina, Sars 1866

Superfamily Darwinuloidea Brady \& Norman, 1889

Family Darwinulidae Brady \& Norman, 1889

Genus Darwinula Brady \& Robertson, 1885

Darwinula stevensoni (Brady \& Norman, 1870) ${ }^{\text {PHR }}$

Superfamily Cytheroidea Baird, 1850

Family Limnocytheridae Klie, 1938

Sub-family Limnocytherinae Klie, 1938

Genus Leucocythere Kaufmann, 1900

Leucocythere baltica (Diebel, 1965) ${ }^{\mathrm{P}}$

Genus Limnocythere Brady, 1867

Limnocythere falcata Dicbel, $1968^{p}$

Limnocythere inopinata (Baird, 1843) ${ }^{\mathrm{PII}}$ $1869)^{\mathrm{PHR}}$

Limnocythere sanctipatricii (Brady \& Robertson,

Limnocythere stationis Vavra, $1891^{\mathrm{P}}$

Limnocythere cf. usenensis Karmischina, 1970

Genus Paralimnocythere Carbonnel, 1965.

Paralimnocythere compressa (Brady \& Norman, 1889) PIIR $^{\mathrm{P}}$

Paralimnocythere cf. diebeli (Petkovski, 1969) Diebel \& Pietrzeniuk, $1978^{\mathrm{P}}$

Paralimnocythere relicta (Lilljeborg, 1863) ${ }^{\mathrm{R}}$

Sub-family Timiriaseviinae Mandelstam, 1960

Genus Metacypris Brady \& Robertson, 1870

Metacypris cordata Brady \& Robertson, $1870^{\mathrm{PHR}}$

Family Cytherideidae Sars, 1925

Sub-family Cytherideinae Sars, 1925

Genus Cytherissa (Sars, 1863)

Cytherissa lacustris (Sars, 1863) ${ }^{\mathrm{PHR}}$

Genus Cyprideis (Jones, 1850)

Cyprideis torosa (Jones, 1850$)^{\mathrm{PHR}}$

Superfamily Cypridoidea Baird, 1845

Family Candonidae Kaufmann, 1900

Sub-family Candoninae Kaufmann, 1900

Genus Candona s.s. Baird, 1845

Candona angulata G. W. Müller, $1900^{\mathrm{PR}}$

Candona brevicornis Klie, $1925^{\mathrm{H}}$

Candona candida (O. F. Müller, 1776) PHR $^{\mathrm{HR}}$

? Candona lactea Baird, $1850^{\mathrm{R}}$

Candona levanderi Hirschmann, $1912^{\mathrm{P}}$

? Candona lozeki Absolon, $1973^{\mathrm{PH}}$

Candona neglecta Sars, $1887^{\mathrm{PI} 1 \mathrm{R}}$

Candona tricicatricosa Dicbel \& Pietrzeniuk, 1969

Genus Cryptocandona Kaufmann, 1900

Cryptocandona reducta $(\mathrm{Alm}, 1913)^{\mathrm{R}}$

Cryptocandona vavrai Kaufmann, $1900^{\mathrm{HR}}$

Genus Fabaeformiscandona Krstic, 1972

Fabaeformiscandona acuminata (Fischer, 1854) ${ }^{\mathrm{R}}$

Fabaeformiscandona balatonica (Daday, 1894) ${ }^{\mathrm{PH}}$

Fabaeformiscandona caudata (Kaufmann, 1900) ${ }^{\mathrm{HR}}$

Fabaeformiscandona fabella (Nüchterlein, 1969) ${ }^{\mathrm{H}}$

Fabaeformiscandona fabaeformis (Fischer, 1851) PHR $^{\mathrm{PH}}$

Fabaeformiscandona fragilis (Hartwig, 1898) ${ }^{\mathrm{R}}$

$1870)^{\mathrm{R}}$

Fabaeformiscandona hyalina (Brady \& Robertson,

Fabaeformiscandona protzi (Hartwig, 1898) ${ }^{\mathrm{PR}}$

Fabaeformiscandona siliquosa (Brady, 1910) ${ }^{\mathrm{R}}$

Genus Pseudocandona Kaufmann, 1900

Pseudocandona albicans (Brady, 1864) ${ }^{\mathrm{PHI}}$

Pseudocandona breuili (Paris, 1920) ${ }^{\mathrm{H}}$
Pseudocandona compressa (Koch, 1838) PH $^{\mathrm{H}}$

Pseudocandona cf. eremita (Vejdovsky, 1882) ${ }^{\mathrm{H}}$

Pseudocandona insculpta (G. W. Müller, 1900) ${ }^{\mathrm{R}}$

Pseudocandona lobipes (Hartwig, 1900) ${ }^{\mathrm{HR}}$

Pseudocandona marchica (Hartwig, 1899) ${ }^{\mathrm{PII}}$

Pseudocandona pratensis (Hartwig, 1901) PlIR $^{\mathrm{PI}}$

Pseudocandona rostrata (Brady \& Norman, 1889$)^{\mathrm{H}}$

Pseudocandona sarsi (Hartwig, 1899) ${ }^{\mathrm{R}}$

Pseudocandona stagnalis (Sars, 1890$)^{\mathrm{R}}$

Genus Candonopsis Vávra, 1891

Candonopsis kingsleii (Brady \& Robertson, 1870) ${ }^{\mathrm{PHR}}$

Candonopsis scourfieldi Brady, $1910^{\mathrm{R}}$

Genus Nannocandona Ekman, 1914

Nannocandona faba Ekman, $1914^{\mathrm{Pl}}$

Genus Paracandona Hartwig, 1899

Paracandona euplectella (Robertson, 1889) ${ }^{\mathrm{HR}}$

Sub-family Cyclocypridinae Kaufmann, 1900

Genus Cyclocypris Brady \& Norman, 1889 Cyclocypris globosa (Sars, 1863) ${ }^{\mathrm{R}}$

Cyclocypris laevis (O. F. Müller, 1776) PHR $^{\mathrm{PHR}}$

Cyclocypris ovum (Jurine, 1820) ${ }^{\mathrm{PHR}}$

Cyclocypris serena (Koch, 1838) ${ }^{\mathrm{P} 1 \mathrm{R}}$

Genus Cypria (Zenker, 1854)

Cypria exsculpta Fischer, $1855^{\mathrm{R}}$

Cypria ophtalmica (Jurine, 1820) PHR $^{\mathrm{R}}$

Family Ilyocyprididae Kaufmann, 1900)

Genus Ilyocypris Brady \& Norman, 1889

Iyocypris biplicata Koch, $1838^{\mathrm{PH}}$

Ilyocypris bradyi Sars, $1890^{\mathrm{PIR}}$

Ilyocypris decipiens Masi, $1905^{\mathrm{R}}$

Ilyocypris getica Masi, $1906^{\mathrm{R}}$

Ilyocypris gibba (Ramdohr, 1808) ${ }^{\mathrm{PHR}}$

Ilyocypris inermis Kaufmann, $1900^{\mathrm{PHR}}$

Ilyocypris lacustris Kaufmann, $1900^{\mathrm{P}}$

Iyocypris monstrifica (Norman, 1862) PR $^{\mathrm{PR}}$

Ilyocypris papillata Robinson, $1990^{\mathrm{P}}$

Ilyocypris quinculminata Sylvester-Bradley, $1973^{\mathrm{P}}$

Ilyocypris schwarzbachi Kempf, 1967 ${ }^{\mathrm{P}}$

Family Notodromatidae Kaufmann, 1900

Sub-family Notodromatinae Kaufmann, 1900

Genus Notodromas Lilljeborg, 1853

Notodromas monacha (O. F. Müller, 1776) ${ }^{\mathrm{R}}$

Sub-family Cyproidinae Hartmann, 1963

Genus Cyprois Zenker, 1854

Cyprois marginata (Straus, 1821) $\mathrm{PHR}$

Family Cyprididae Baird, 1845

Sub-family Cypricercinae McKenzie, 1971

Genus Bradleystrandesia Broodbakker, 1983

Bradleystrandesia fuscata (Jurine, 1820) $\mathrm{HR}$

Bradleystrandesia reticulata (Zaddach, 1844) ${ }^{\mathrm{IR}}$

Genus Strandesia Stuhlmann, 1888

Strandesia obliqua (Brady, 1868) (HK $^{\mathrm{H}}$

Sub-family Cypridinae Baird, 1845

Genus Cypris O. F. Müller, 1776

Cypris bispinosa Lucas, $1849^{\mathrm{R}}$

Cypris pubera O. F. Müller, $1776^{\mathrm{PHR}}$

Sub-family Dolerocypridinac Tricbcl, 1961

Genus Dolerocypris Kaufmann, 1900

Dolerocypris fasciata (O. F. Müller, 1776) ${ }^{\mathrm{R}}$

Sub-family Eucypridinae Bronshtein, 1947

Genus Eucypris (Vávra, 1891)

Eucypris anglica Fox, $1967^{\mathrm{R}}$

Eucypris crassa (O. F. Müller, 1785) ${ }^{R}$

Eucypris dulcifrons Diebel \& Pietrzeniuk, $1969^{\mathrm{P}}$

Eucypris elliptica (Baird, 1846) ${ }^{\mathrm{R}}$ 
Eucypris cf. gemella Bodina, $1961^{p}$ Eucypris heinrichi Diebel \& Pietrzeniuk, $1978^{\mathrm{PHI}}$ Eucypris lilljeborgi (G. W. Müller, 1900) $)^{\mathrm{IIR}}$ Eucypris ornata (O. F. Müller, 1776) ${ }^{\mathrm{R}}$ Eucypris pigra (Fischer, 1851) PHR $^{\mathrm{PHR}}$ Eucypris virens (Jurine, 1820) PHR $^{\text {PHR }}$

Genus Prionocypris Brady \& Norman, 1896 Prionocypris serrata (Norman, 1861) $\mathrm{PHR}$

Genus Tonnacypris Diebel \& Pietrzeniuk, 1975 Tonnacypris lutaria (Koch, 1838) ${ }^{\mathrm{HR}}$

Genus Trajancypris Martens, 1989

Trajancypris clavata (Baird, 1838) ${ }^{\mathrm{PIR}}$

? Trajancypris serrata (G. W. Müller, 1900) ${ }^{\mathrm{R}}$

Sub-family Herpetocypridinae Kaufmann, 1900

Genus Herpetocypris Brady \& Norman, 1889

Herpetocypris brevicaudata Brady \& Norman, $1889^{\mathrm{PHR}}$

Herpetocypris chevreuxi Sars, $1896^{\mathrm{PHR}}$

? Herpetocypris ehringsdorfensis Diebel \& Wolfschläger,

$1975^{\mathbf{P}}$

Herpetocypris reptans (Baird, 1835) ${ }^{\mathrm{PHR}}$

Genus Psychrodromus Danielopol \& McKenzic, 1977

Psychrodromus olivaceus (Brady \& Norman, 1889) PHR $^{\mathrm{R}}$

Psychrodromus robertsoni (Brady \& Robertson, 1889) ${ }^{\mathrm{R}}$

Genus Stenocypria G. W. Muiller, 1901

Stenocypria fischeri (Lilljeborg, 1883) ${ }^{\mathrm{H}}$

Sub-family Scottinae Bronshtein, 1947

Genus Scottia Brady \& Norman, 1889

Scottia browniana (Jones, 1850$)^{\mathrm{P}}$

Scottia pseudobrowniana Kempt, 1971 ${ }^{\mathrm{PHR}}$

Scottia tumida Kempf, $1971^{\mathrm{P}}$

Sub-family Cyprinotinae Bronshtein, 1947

Genus Heterocypris Claus, 1892

Heterocypris incongruens (Ramdohr, 1808) ${ }^{\mathrm{HR}}$

Heterocypris salina (Brady, 1868) ${ }^{\mathrm{PHR}}$

Sub-family Isocypridinae Rome, 1965

Genus Isocypris G. W. Müller. 1908

Isocypris beauchampi (Paris, 1920) ${ }^{\mathrm{K}}$

Sub-family Cypridopsinae Kaufmann, 1900

Genus Cypridopsis Brady, 1867

Cypridopsis hartwigi G. W. Müller, $1900^{\mathrm{PHR}}$

Cypridopsis lusatica Schäffer, $1943^{R}$

Cypridopsis obesa Brady \& Robertson, $1869^{\mathrm{R}}$

Cypridopsis vidua (O. F. Müller, 1776) PHR $^{\mathrm{PH}}$

Genus Plesiocypridopsis (Rome, 1965)

Plesiocypridopsis newtoni (Brady \& Robertson, 1870) ${ }^{\mathrm{HR}}$

Genus Sarscypridopsis McKcnzic, 1977

Sarscypridopsis aculeata (Costa, 1847) $\mathrm{HR}$

Genus Cavernocypris Hartmann, 1964

Cavernocypris subterranea (Wolf, 1920) HR $^{\mathrm{HR}}$

Genus Potamocypris Brady, 1870

Potamocypris arcuata (Sars, 1903) ${ }^{\text {PHR }}$

Potamocypris fallax Fox, $1967^{\mathrm{HR}}$

Potamocypris fulva (Brady, 1868) PHR $^{\mathrm{PH}}$

Potamocypris pallida Alm, $1914^{\mathrm{R}}$

Potamocypris similis G. W. Müller, $1912^{\mathrm{HR}}$

Potamocypris smaragdina (Vávra, 1891) ${ }^{\mathrm{HR}}$

Potamocypris variegata (Brady \& Norman, 1889)

Potamocypris villosa (Jurine, 1820$)^{\mathrm{PHR}}$

Potamocypris zschokkei (Kaufmann, 1900) PHR $^{\text {PR }}$

\section{ACKNOWLEDGEMENTS}

Our thanks to Dr S. Halsey (Crustacea Section, BMNH) for allowing our examination of type material from the Museum's collections. Professor T. K. Petkovski (Macedo- nian Museum of Natural History, Skopje), Dr D. J. Horne (University of Greenwich, London), Dr P. A. Henderson (National Power Marine Biology Labs, Fawley) and Dr C. Meisch (National Museum of Natural History, Luxembourg) provided valuable discussions and advice. Dr P. Marmonier (University of Savoy) and Dr J. E. Robinson (University College, London) are thanked for comparative material. Special thanks to Dr K. Martens (KBIN, Brussels) who kindly read through an earlier version of this manuscript, made many useful comments and criticisms, and also provided a preprint of his study of Paralimnocythere. Thanks also to our many colleagues for their ready help, advice, and assistance.

This study was funded by a SERC research grant to J. G. Evans.

\section{Manuscript received August 1992 Manuscript accepted May 1993}

\section{REFERENCES}

Bowman, T. E. \& Abele, L. G. 1982. Classification of the Recent Crustacea. In Abele, L. G. (Ed.), The biology of Crustacea, volume 1. Systematics, the fossil record and evolution, 1-27, Academic Press, New York.

Brady, G. S. 1910. A revision of the British species of ostracod Crustacea belonging to the subfamilies Candoninae and Herpetocypridinae, (with a note on a parasitic worm, by Miss M.V. Lebour, M.Sc.). Proceedings of the Zoological Society of London, London, 1910: 194-220, pls 19-30.

Brady, G. S. \& Norman, A. M. 1889. A monograph of the marine and freshwater Ostracoda of the North Atlantic and of North-western Europe. Section I. Podocopa. Scientific Transactions of the Royal Dublin Society, Dublin, 4: 63-270, pls 8-23.

Brady, G. S. \& Robertson, D. 1869. Notes on a week's dredging in the west of Ireland. Annals and Magazine of Natural History London, Ser. 4, 3: 353-374, pls 18-22.

Broodbakker, N. W. 1983. The genus Strandesia and other Cypricercini (Crustacea, Ostracoda) in the West Indies. Part 1. Taxonomy. Bijdragen tot de Dierkunde, Leiden, 53: 327--368.

Carbonel, P., Colin, J. P., Danielopol, D. L., Löffler, H. \& Neustrueva, I. 1988. Paleoecology of limnic ostracodes: a revicw of some major topics. Palaeogeography, Palaeoclimatology, Palaeoecology, Amsterdam, 62: 413-461.

Carbonnel, G. 1965. Sur un nouveau genre (Paralimnocythere) et une nouvelle espèce ( $P$. bouleigensis) d'ostracodes du Tortonien. Archives des Sciences, Genève, Geneva, 18: 146-150, pls 1-2.

Catt, J. A. \& Penny, L. F. 1962. The Pleistocene deposits of Holderness, East Yorkshire. Proceedings of the Yorkshire Geological Society, Leeds, 35: 375-420.

Colin, J. P. \& Danielopol, D. L. 1980. Sur la morphologic, la systématique, la biogéographie et l'évolution des ostracodes Timiriaseviinae (Limnocytheridae). Paléobiologie continentale, Montpellier, 11: 1-51, pls 1-16.

Danielopol. D. L. 1978. Über Herkunft und Morphiogie der Süsswasser-hypogäischen Candoninae (Crustacea, Ostracoda). Sitzungsberichte der Österreichischen Akademie der Wissenschaften, Mathematisch-naturwissenschaftliche Klasse, Abteilung 1, Vienna, 187: 1-162.

Danielopol, D. L. 1982. Nouvelles donées sur les Candoninae (Ostracoda) hypogés de Roumanie et Yugoslavie. Bulletin du Museum national d'Histoire naturelle de Paris, (Sect. A)., Paris, 4: 369-396.

Danielopol, D. L. \& McKenzic, K. G. 1977. Psychrodromus gen. n. (Crustacca, Ostracoda), with redescription of the cypridid genera Prionocypris and Ilyodromus. Zoologica Scripta, Stockholm, 6: 301-322.

Danielopol, D. L., Martens, K. \& Casale, L. M. 1989. Revision of the genus Leucocythere Kaufmann, 1892 (Crustacea, Ostracoda, Limnocytheridae), with the description of a new species. Bulletin 
van het koninklijk Belgisch Instituut voor Natuurwetenschappen, Biologie, Brussels, 59: 63-94.

De Deckker, P. \& Forester, R. M. 1988. The use of ostracods to reconstruct continental palaeoenvironmental records. In De Deckker, P., Colin, J. P. \& Peypouquet, J. P. (Eds), Ostracoda in the Earth Sciences, 175-199 Elsevier Science Publishers, Amsterdam.

Delorme, L. D. 1970. Freshwater ostracodes of Canada. Part 4. Families Ilyocyprididae, Notodromadidae, Darwinulidae, Cytherideidae and Entocytheridae. Canadian Journal Zoology, Ottowa, 48: $1251-1259$, pls 1-6.

Delorme, L. D. 1989. Methods in Quaternary ecology No 7. Freshwater ostracodes. Geoscience Canada, St. John's, 16: 85-90.

Diebel, K. \& Pietrzeniuk, E. 1969. Ostracoden aus dem Mittelplcistozän von Süssenborn bei Weimar. Paläontologische Abhandlungen, Abteilung A, Paläozoologie, Berlin 3: 463-488, pls $7-10$.

Diebel, K. \& Pictrzeniuk, E. 1978. Die Ostrakodenfauna aus den Jungpleistozänen (weichselkaltzeitlichen) Deckschichten von Burgtonna in Thüringen. Quartärpaläontologie, Berlin, 3: 207-221, pls 49-53.

Diebel, K. \& Pictrzeniuk, E. 1984. Jungpleistozäne Ostrakoden aus Scdimenten der Parkholen von Weimar. Quartärpaläontologie, Bcrlin, 5: 285-319, pls 1-10.

Douglas, D. J. \& Healy, B. 1991. The freshwater ostracods of two quagmires in Co. Louth, Ireland. Verh. Internat. Verhandlungen der Internationalen Vereinigung für theoretische und angewandte Limnologie, Stuttgart, 24: 1522-1525.

Evans, J. G. \& Griffiths, H. I. 1993. Holocene mollusc and ostracod sequences; their potential for examining short-timescale evolution. In Lees, D. R. \& Edwards, D. (Eds), Evolutionary Patterns and Processes, 125-137 Academic Press, London for Linnean Society of London.

Fox, H. M. 1967. More new and interesting cyprids (Crustacea, Ostracoda) from Britain. Joumal of Natural History, London, 4: 549-559.

Fuhrmann, R. (1991). Ostrakoden aus den HolsteinInterglazialbecken Wildschütz und Dahlen (Sachsen). Zeitschrift für Geologische Wissenschaften, Berlin, 19: 269-288, pls 1-3.

Gibbard, P. L., Coope, G. R., Hall, A. R., Preece, R. C. \& Robinson, J. E. 1981. Middle Devensian deposits beneath the 'Upper Floodplain' terrace of the River Thames at Kempton Park, Sunbury, England. Proceedings of the Geologists' Association, London, 93: 275-289.

Griffiths, H. I. \& Evans, J. G. 1992. Potamocypris arcuata (Sars, 1903) (Ostracoda) new to Britain. Crustaceana, Leiden, 62: $110-112$.

Griffiths, H. I. \& Mount, R. 1993. Ostracods. In Evans, J.G. Limbrey, S., Mount, R. \& Máté, I., An environmental history of the Upper Kennet Valley, Wiltshire, for the last 10,000 years Proceedings of the Prehistoric Society, London, 59: 139-195.

Ham, S. F. 1982. The Crustacea of some chalk streams in southern England. Hydrobiologia, Dordrecht, 97: 193-201.

Hartmann, G \& Puri, H. S. 1974. Summary of neontological and palaeontological classification of Ostracoda. Mitteilungen aus dem Hamburgischen Zoologischen Museum und Institut, Hamburg, 70: $7-73$.

Henderson, P. A. 1986. Cypridopsis bamberi sp. nov., a new species of ostracod (Crustacea, Podocopida) from England. Journal of Natural History, London, 20: 1-5.

Henderson, P. A. 1990. Freshwater Ostracods. Synopses of the British Fauna (New Series), 42:. Universal Book Services/Dr W. Backhuys, Oestgeest for Linnean Society of London and Estuarine and Coastal Sciences Association.

Holmes, J. A. 1992. Non-marine ostracods as Quaternary palaeoenvironmental indicators. Progress in Physical Geography, Seven Oaks, 16: 405-431.

Holmes, P. F. 1937. Pseudocandona elongata, a new species of ostracod. Annals and Magazine of Natural History, (Ser. 10), London, 19: 422-430.

Horne, D. J., Horne, D. M. \& Horne, J. E. M. 1990. New records of ostracods from the Lake District. Transactions of the Natural
History Society of Northumbria, Newcastle-upon-Tyne, 55: $147-148$

Keen, D. H., Jones, R. L. \& Robinson, J. E. 1984. A late Devensian and early Flandrian fauna and flora from Kildale, north-east Yorkshire. Proceedings of the Yorkshire Geological Society, Leeds, 44: 385-397.

Kempf, E. K. 1971. Ökologie, Taxonomie und Verbreitung der nichtmarinen Ostracoden-Gattung Scottia im Quartär von Europe. Eiszeitalter und Gegenwart, Öhringen, 22: 43-63.

Kempf, E. K. 1980a. Index and bibliography of nonmarine Ostracoda. 1. Index A. Sonderveröffentlichungen der Geologischen Institut der Universität zu Köln, Cologne, 35: 1-188.

Kempf, E. K. 1980b. Index and bibliography of nonmarine Ostracoda. 4. Bibliography A. Sonderveröffentlichnngen der Geologischen Institut der Universität zu Köln, Cologne, 38: 1-186.

Klie, W. 1938. Krebstiere oder Crustacea, III: Ostracoda, Muschelkrebse. Die Tierwelt Deutschlands und der angrenzenden Meeresteile nach ihren Merkmalen und nach ihrer Lebensweise 34. Gustav Fischer Verlag, Jena.

Maitland, P. S. 1977. A coded check-list of animals occurring in fresh water in the British Isles, Institute of Terrestrial Ecology, Edinburgh.

Marmonier, P. \& Danielopol, D. L. 1988. Découverte de Nannocandona faba Eckman (Ostracoda, Candonine) en basse Autriche. Son origine et son adaptation au milieu interstitiel. Vie Milieu, Paris, 38: 35-38.

Marmonier, P., Meisch, C. \& Danielopol, D. L. 1989. A review of the genus Cavernocypris Hartmann (Ostracoda, Cypridopsinae): systematics, ecology and biogeography. Bulletin de la Société des Naturalistes Luxembourgeois, Luxembourg, 89: 221-278.

Martens, K. 1989. On the systematic position of the Eucypris clavata-group, with a description of Trajancypris gen. nov. (Crustacea, Ostracoda). Archiv für Hydrobiologie, Stuttgart, 83(S): $227-251$.

Martens, K. 1990. Speciation and evolution in the genus Limnocythere Brady, 1867 sensu stricto (Crustacea, Ostracoda), in the east African Galla and Awassa Basins (Ethiopia). Courier Forschungs-Institut Senckenberg, Frankfurt, 123: 87-95.

Martens, K. 1991. On a small collection of non-marine ostracods from Mongolia, with the description of a new species. Miscellanea zoologica Hungarica, Bucharest, 6: 53-60.

Martens, K. 1992. A reassessment of Paralimnocythere Carbonnel, 1965 (Crustacea, Ostracoda, Limnocytherinae), with a description of a new genus and two new species. Bulletin van het Koninklijk Belgisch Instituut voor Natuurwetenschappen, Biologie, Brussels, 62: $125-168$

Meisch, C. 1984. Revision of the Recent western Europe species of genus Potamocypris (Crustacea, Ostracoda). Part 1. Species with short swimming setae on the second antennae. Travaux Scientifique du Museum national d'Histoire naturelle de Luxembourg, Luxembourg, 3: 1-55.

Meisch, C. 1985. Revision of the Recent West European species of the genus Potamocypris (Crustacea, Ostracoda), Part 2. Species with long swimming setae on the second antennae. Travaux Scientifique du Museum national d'Histoire naturelle de Luxembourg, Luxembourg, 6: 1-96.

Meisch, C. 1988. Ostracodes récoltés à Paris. Avec une clef pour la détermination des espèces européennes du genre Ilyocypris (Crustacea, Ostracoda). Bulletin de la Société des Naturalistes Luxembourgeois, Luxembourg, 88: 145-163.

Meisch, C. 1991. Revision of the freshwater ostracod species Cypridopsis hartwigi and Cypridopsis elongata. With a generic key to the European Cypridopsinae (Crustacea, Ostracoda). Bulletin de la Société des Naturalistes Luxembourgeois, Luxembourg, 92: $159-178$.

Meisch, C., Wouters, K. \& Martens, K. 1990. Liste annotée des ostracodes actuels non-marins trouvés en France. Travaux Scientifique $d u$ Museum national d'Histoire naturelle de Luxembourg, Luxembourg, 15: 1-62.

Mount, R. 1991. An environmental history of the Upper Kennet River Valley and some implications for human communities. Unpublished Ph.D. thesis, University of Wales. 
Norman, A. M. 1905. Irish Crustacea Ostracoda. Irish Naturalist, Dublin, 16: $137-155$.

Nüchterlein, H. 1969. Süsswasserostracoden aus Franken. Ein beitrag, zur Systematik und Ökologie der Ostracoden. Internationale Revue der gesamten Hydrobiologie, Berlin, 54: $223-287$.

Paris, P. 1920. Biospeologica. XLI. Ostracodes (première sćrie). Archives de Zoologie experimentale et génerale, Paris, 58: 475-487, pls $18-21$.

Petkovski, T. K. 1963. Über Süsswasser-ostracoden der Azoren. Boletin do Museu Municipal do Funchal, Funchal, 17: 49-65.

Petkovski, T. K. 1969. Limnocythere-Arten aus Mazedonien (Crustacea, Ostracoda). Acta Musei Macedonici Scientiarum Naturalium, Skopjc, 12(1): 1-18, 1 pl.

Petkovski, T. K., Meisch, C. \& Wouters. K. 1993. Taxonomic revision of the freshwater ostracod species Cypridopsis lusatica Schäfer, 1943 (Crustacea). Travaux Scientifique du Museum national d'Histoire naturelle de Luxembourg, Luxembourg, 19: 49-66.

Preece, R.C. \& Robinson, J. E. 1984. Late Devensian and Flandrian environmental history of the Ancholme Valley, Lincolnshire: molluscan and ostracod evidence. Journal of Biogeography, London, 11: 319-352.

Prece, R. C., Coxon, P. \& Robinson, J. E. (1986). New biostratigraphic evidence of the Post-glacial colonization of Ireland and for Mesolithic forest disturbance. Journal of Biogeography, London, 13: 487-509.

Robinson, E. 1978. The Pleistocene. In Bate, R. H. \& Robinson, E.
(Eds), A Stratigraphical Index of British Ostracoda, 452-472, Seel House Press, Liverpool.

Robinson, J. E. 1990. The ostracod fauna of the Middle Pleistocene interglacial deposits at Little Oakley, Essex. Philosophical Transactions of the Royal Society of London. Ser. B., London. 328: $409-423$.

Scourfield, D. J. 1904. Synopsis of the known species of British freshwater Entomostraca. Part III Ostracoda, Phyllopoda and Branchiura. Joumal of the Quicket Microscopical Club. London. 9: $29-44$.

Sywula, T. 1974. Malzoraczki (Ostracoda). Fauna Slodkowodna Polski 24: Polska Akademia Nauk, Warsaw.

Van Frausum, A. \& Wouters. K. 1990. Ostracoda from Holocene calcareous tufa deposits in southern Belgium: a palacocnvironmental analysis. In Whatley, R. \& Maybury, C. (Eds). Ostracoda and global events, 505-511. Chapman \& Hall, London.

Van Harten, D. 1979. Some new shell characters to diagnose the species of the llyocypris gibba-biplicata-bradyi group and their ecological significance. In Krstic, N. (Ed.), Taxonomy, Biostratigraphy \& Distribution of Ostracodes, 71-76, pls 1-2, Scrbian Geological Society, Belgrade.

Willing, M. J. 1985. The biostratigraphy of Flandrian tufa deposits in the Cotswold and Mendip districts. Unpublished Ph.D. thesis, University of Sussex.

Wouters, K. 1989. Check-list of the recent non-marine Ostracoda (Crustacea) of Belgium. In Wouters, K. \& Beart, L. (Eds), Verhandlungen van het symposium 'Invertebraten van Belgie', 153-158, Koninklijk Belgisch Instituut voor Natuurwetenschappen, Brussels. 